University of Nebraska - Lincoln

DigitalCommons@University of Nebraska - Lincoln

US Department of Energy Publications

U.S. Department of Energy

2009

\title{
Morphology and Deformation Mechanisms and Tensile Properties of Tetrafunctional Multigraft Copolymers
}

\author{
Yongxin Duan \\ Institute of Materials Science and Technology (IMT), Friedrich-Schiller-University Jena, Lobdergraben 32, \\ D-07743 Jena, Germany \\ Mahendra Thunga \\ Institute of Materials Science and Technology (IMT), Friedrich-Schiller-University Jena, Lobdergraben 32, \\ D-07743 Jena, Germany \\ Ralf Schlegel \\ Institute of Materials Science and Technology (IMT), Friedrich-Schiller-University Jena, Lobdergraben 32, \\ D-07743 Jena, Germany \\ Konrad Schneider \\ Leibniz-Institute of Polymer Research Dresden, Hohe Strasse 6, D-01069 Dresden, Germany \\ Erik Rettler \\ Department of Physical Chemistry, University of Duisburg-Essen, Schutzenbahn 70,D45117 Essen, \\ Germany \\ Seef nextpage for additional authors and and adtional works at:htigitalcommons.unl.edu/usdoepub \\ Part of the Bioresource and Agricultural Engineering Commons
}

Duan, Yongxin; Thunga, Mahendra; Schlegel, Ralf; Schneider, Konrad; Rettler, Erik; Weidisch, Roland; Siesler, Heinz W.; Stamm, Manfred; Mays, Jimmy W.; and Hadjichristidis, Nikos, "Morphology and Deformation Mechanisms and Tensile Properties of Tetrafunctional Multigraft Copolymers" (2009). US Department of Energy Publications. 69.

https://digitalcommons.unl.edu/usdoepub/69

This Article is brought to you for free and open access by the U.S. Department of Energy at DigitalCommons@University of Nebraska - Lincoln. It has been accepted for inclusion in US Department of Energy Publications by an authorized administrator of DigitalCommons@University of Nebraska - Lincoln. 


\section{Authors}

Yongxin Duan, Mahendra Thunga, Ralf Schlegel, Konrad Schneider, Erik Rettler, Roland Weidisch, Heinz W. Siesler, Manfred Stamm, Jimmy W. Mays, and Nikos Hadjichristidis 


\title{
Morphology and Deformation Mechanisms and Tensile Properties of Tetrafunctional Multigraft Copolymers
}

\author{
Yongxin Duan,,${ }^{\dagger \neq, \|}$ Mahendra Thunga, ${ }^{\dagger,}$ Ralf Schlegel, ${ }^{\dagger}$ Konrad Schneider, ${ }^{\dagger}$ Erik Rettler, ${ }^{\S}$ \\ Roland Weidisch, $,{ }^{\dagger}, *$ Heinz W. Siesler, ${ }^{\S}$ Manfred Stamm, ${ }^{\S}$ Jimmy W. Mays," and \\ Nikos Hadjichristidis ${ }^{\perp}$
}

\begin{abstract}
$\dagger$ Institute of Materials Science and Technology (IMT), Friedrich-Schiller-University Jena, Lobdergraben 32, D-07743 Jena, Germany, 'Leibniz-Institute of Polymer Research Dresden, Hohe Strasse 6, D-01069 Dresden, Germany, ${ }^{\S}$ Department of Physical Chemistry, University of Duisburg-Essen, Schutzenbahn 70, D 45117 Essen, Germany, "Department of Chemistry, University of Tennessee, Knoxville, Tennessee 37996, and Chemical Sciences Division and Center for Nanophase Materials Sciences, Oak Ridge National Laboratory, Oak Ridge, Tennessee 37831, ${ }^{\perp}$ Department of Chemistry, University of Athens, Athens, Greece, and ${ }^{\#}$ Key Laboratory of Rubber-Plastics (QUST), Ministry of Education, Qingdao University of Science and Technology, Zhengzhou Road, Qingdao 266042, China
\end{abstract}

Received February 24, 2009; Revised Manuscript Received April 24, 2009

\begin{abstract}
Morphology and deformation mechanisms and tensile properties of tetrafunctional multigraft (MG) polystrene-g-polyisoprene (PS- $g$-PI) copolymers were investigated dependent on PS volume fraction and number of branch points. The combination of various methods such as TEM, real time synchrotron SAXS, rheo-optical FTIR, and tensile tests provides comprehensive information at different dimension levels. TEM and SAXS studies revealed that the number of branch points has no obvious influence on the microphase-separated morphology of tetrafunction MG copolymers with $16 \mathrm{wt} \%$ PS. But for tetrafunctional MG copolymers with $25 \mathrm{wt} \%$ PS, the size and integrity of PS microdomains decrease with increasing number of branch point. The deformation mechanisms of MG copolymers are highly related to the morphology. Dependent on the microphase-separated morphology and integrity of the PS phase, the straininduced orientation of the PS phase is at different size scales. Polarized FT-IR spectra analysis reveals that, for all investigated MG copolymers, the PI phase shows strain-induced orientation along SD at molecular scale. The proportion of the PI block effectively bridging PS domains controls the tensile properties of the MG copolymers at high strain, while the stress-strain behavior in the low-mediate strain region is controlled by the continuity of PS microdomains. The special molecular architecture, which leads to the higher effective functionality of PS domains and the higher possibility for an individual PI backbone being tethered with a large number of PS domains, is proposed to be the origin of the superelasticity for MG copolymers.
\end{abstract}

\section{Introduction}

Multigraft (MG) polystyrene-g-polyisoprene (PS-g-PI) copolymers with various molecular architectures, as shown in Figure 1, are novel thermoplastic elastomers (TPE) which were synthesized successfully by Mays et al. ${ }^{1-6}$ This special synthetic route provides a method to design and control the molecular weight of the backbone and the graft arm as well as the number of branch points and the number of arms grafted to each junction point. It has been shown that the morphological behavior of these multigraft copolymers can be predicted by the model of Milner on the basis of the constituting block copolymer concept. ${ }^{7}$ Preliminary studies have reported that MG copolymers with $16-25$ wt $\%$ PS possess exceptional elasticity compared to commercial TPE $;^{4-6}$ i.e., they can be stretched up to about $1400 \%$ with a residual strain of only $40 \%$ after unloding, which was observed for the first time in the field of TPEs. It has been found that the architectural parameters which significantly influence the morphology and mechanical behavior of MG copolymers are the functionality of the branch points and the number of the branch points per molecule.

In spite of a very good application prospect, the structureproperty correlations and deformation mechanisms of $\mathrm{MG}$ copolymers are still unclear. For example, although all of the

\footnotetext{
*Corresponding author: e-mail roland.weidisch@uni-jena.de,
} Fax +493641947702 .
MG copolymers with 16-25 wt \% PS content show exceptionally high strain at break, the modulus and tensile strength are strongly dependent on the molecular parameters such as functionality and number of branch points. This provides a very wide spectrum to tailor material properties without change of the PS content. But what is the dominant factor accounting for the exceptionally high ultimate strain? Besides the molecular architecture parameters, how does the morphology influence mechanical properties? To understand thoroughly the nature of superelasticity and clarify the deformation mechanism of MG copolymers, it is necessary to monitor the structural changes of materials during the deformation process at different dimension scales. SAXS and rheo-optical FT-IR are very powerful techniques to investigate the deformation behavior of materials.

SAXS is the most widely used technique to study the deformation mechanism of multiphase-separated copolymers. ${ }^{8-17}$ The pioneering work of Hashimoto et al..$^{8-10}$ investigated the deformation behavior of sphere forming SIS and cylinder-forming SBS copolymers. It was found that the deformation of sphere-forming copolymers deviates from affine behavior at quite small strain which is caused by the formation of cavities. The study on SBS copolymers with cylindrical PS microdomains suggested that the deformation at early and intermediate stages is controlled by the inherent morphology, while at large extension the deformation and resulting structure are determined by molecular orientation 
(a)

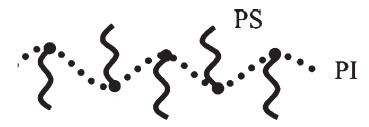

(b)

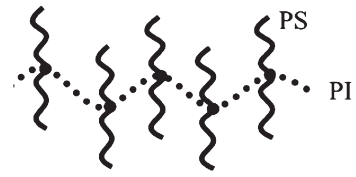

(c)

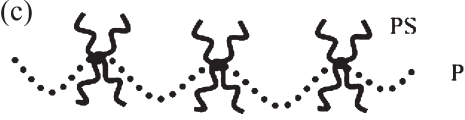

Figure 1. Illustration of multigraft copolymers based on PI backbone and PS branches with regularly spaced (a) trifunctional, (b) tetrafunctional, and (c) hexafunctional junction points.

in the rubber phase. In addition, they illustrated a systematic variation in SAXS pattern with respect to the orientation of PS microdomains. The PS domains assuming a preferential orientation inclined to the stretching direction give rise to a four-point pattern. In the publication of Séguéla, and Prud'homme, ${ }^{15-17}$ hydrated SBS triblock copolymers with deferent morphologies like cylindrical and spherical PS microdomains were investigated. For the samples with cylindrical morphology the results are similar to those of Hashimoto et al., whereas the samples with spherical PS domains show different deformation behavior.

The IR technique offers a great advantage for studying the molecular orientation of the multicomponent system because it is possible to analyze the polarization behavior of infrared absorption bands of different components. Since the orientation behavior with strain is closely related to the changes in the microdomain structures, the studies of the molecular orientation by IR can be applied for the investigation of the deformation mechanism. Zhao ${ }^{18}$ was probably the first to discuss the molecular orientation in terms of the structural changes with strain. He studied the structural relaxation in the stretched SBS copolymers with cylindrical microdomains of PS. However, this study was limited to the orientation of the PB phase. In fact, most of the studies concerning the orientation of PS-based block copolymers discussed only the orientation of the soft block chains. ${ }^{19,20}$ But Sakurai et al. studied the strain-induced molecular orientation of SBS copolymers with different morphology and detected the orientation of PS chains. ${ }^{21,22}$ They ascribed the reason why the PS orientation was not detected in most cases to the poor signal-to-noise ratio of the PS absorption bands used to evaluate the degree of orientation.

In one of our previous communications, ${ }^{23}$ the deformation behavior of a trifunctional MG copolymer was examined by synchrotron SAXS and rheo-optical FT-IR at nanometer size scales and molecular level, respectively. On the basis of the finding of the nonaffine deformation and similar orientation behavior of PI and PS segments, it was proposed that the superelasticity is derived from the unique molecular architecture which improves the functionality of PS domains and enhances the stress transfer between PS and PI phases. In this article, the tensile properties of two series of tetrafunctional MG copolymers with 16 and $25 \mathrm{wt} \%$ PS are investigated in detail. The tensile properties at different strain regions (modulus at 300\% elongation, strength and strain at break) are discussed by considering the influence of PS content, number of branch points, and morphology. The corresponding deformation processes are examined by synchrotron SAXS and rheo-optical FT-IR.

\section{Experimental Part}

Details of the synthesis and molecular characterization of MG copolymers were described by Iatrou et al. ${ }^{1-3}$ Molecular
Table 1. Molecular Characteristics of MG Copolymers with Regularly Spaced Junction Points

\begin{tabular}{lccc}
\hline & $M_{\mathrm{w}}\left(\times 10^{3} \mathrm{~g} / \mathrm{mol}\right)$ & $\begin{array}{c}\text { no. of junctions/ } \\
\text { molecule }\end{array}$ & PS (wt \%) \\
\hline MG 4-16-8.5 & 980 & 8.5 & 15.8 \\
MG 4-16-5.1 & 619 & 5.1 & 15.5 \\
MG 4-16-4.2 & 526 & 4.2 & 16.0 \\
MG 4-16-3.3 & 430 & 3.3 & 16.0 \\
MG 4-25-6.6 & 891 & 6.6 & 24.9 \\
MG 4-25-4.4 & 624 & 4.4 & 24.7 \\
MG 4-25-3.2 & 472 & 3.2 & 24.5
\end{tabular}

characteristics of the MG copolymers used in this study are listed in Table 1. A general nomenclature for these multigraft copolymers, MG $n-\phi-\beta$, is employed. MG stands for regular multigraft, and $n$ is the junction point functionality. The $\phi$ represents the total PS weight percent, and the $\beta$ refers to the number of junction points per molecule in a given material, which is sometimes omitted to represent all samples with same functionality and PS content.

Solution-cast films of these MG copolymers were prepared from a nonselective solvent (toluene). The solvent was allowed to evaporate slowly over 7-14 days at room temperature. The films were then dried to a constant weight in a vacuum oven at $120^{\circ} \mathrm{C}$ for 3 days. The thickness of the sample is maintained at $30-40 \mu \mathrm{m}$ for FT-IR studies whereas it is ca. $200 \mu \mathrm{m}$ for SAXS measurements and tensile tests.

Transmission electron microscopy (TEM) studies were performed using a JEOL JEM 3010 operated at an accelerating voltage of $200 \mathrm{kV}$. Ultrathin sections of $40-80 \mathrm{~nm}$ were cut at a sample temperature of $-100{ }^{\circ} \mathrm{C}$ with diamond knives using a Leica Ultracut UCT. These sections were collected on TEM grids and stained in $\mathrm{OsO}_{4}$ vapor for $4 \mathrm{~h}$.

Static SAXS were performed using a homemade three pinhole collimation system with a Rigaku $2 \mathrm{D}$ rotating anode generator $(\mathrm{Cu}$ $\mathrm{K} \alpha$ radiation, $k=0.1542 \mathrm{~nm}$ ), an Osmic multilayer mirror, and a MARCCD detector with a sample-to-detector distance of $1610 \mathrm{~mm}$.

Synchrotron SAXS measurements at room temperature were performed at the Deutsches Elektronen-Synchrotron (DESY) in Hamburg. The beamline BW4 at the DORIS III storage ring at HASYLAB was used. For the measurement

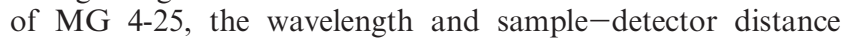
were $0.138 \mathrm{~nm}$ and $7880 \mathrm{~mm}$, respectively. For the measurement of MG 4-16, the wavelength and sample-detector distance were $0.138 \mathrm{~nm}$ and $6049 \mathrm{~mm}$, respectively. A modified stretching device (Kamrath, Weiss) (4,25 $^{2}$ was used to measure SAXS at different strains, which was determined from in situ captured images of the sample during deformation by a digital camera. The design of the stretching machine allows the sample to be uniaxially stretched by equal amounts from the center of the sample, which keeps the center position of the sample fixed in the X-ray beam.

The rheo-optical FT-IR measurements were carried out by employing a computer-controlled electromechanical filmstretching device ${ }^{26}$ mounted in the sample compartment of a Bruker IFS88FT-IR spectrometer. The sample films with gauge dimensions of $7 \times 4 \mathrm{~mm}^{2}$ and a thickness of $30-40 \mu \mathrm{m}$ were uniaxially stretched at a constant strain rate of $3.2 \mathrm{~mm} / \mathrm{min}$. Spectra were measured using alternating parallelly and perpendicularly polarized IR radiation. A pneumatically rotatable wire-grid polarizer (SPECAC) controlled by the computer adjusts the polarization direction of the incident radiation parallel and perpendicular to the stretching direction by a rapid $90^{\circ}$ rotation. The change of the polarization direction is automatically initiated by the last scan of each spectrum. Ten scans per spectrum were accumulated at a spectral resolution of $4 \mathrm{~cm}^{-1}$. A specially designed software ${ }^{27}$ was developed to manage the large number of spectra collected during a rheo-optical experiment.

The tensile tests were performed at a cross-head speed of $15 \mathrm{~mm} / \mathrm{min}$ at room temperature using a universal testing 

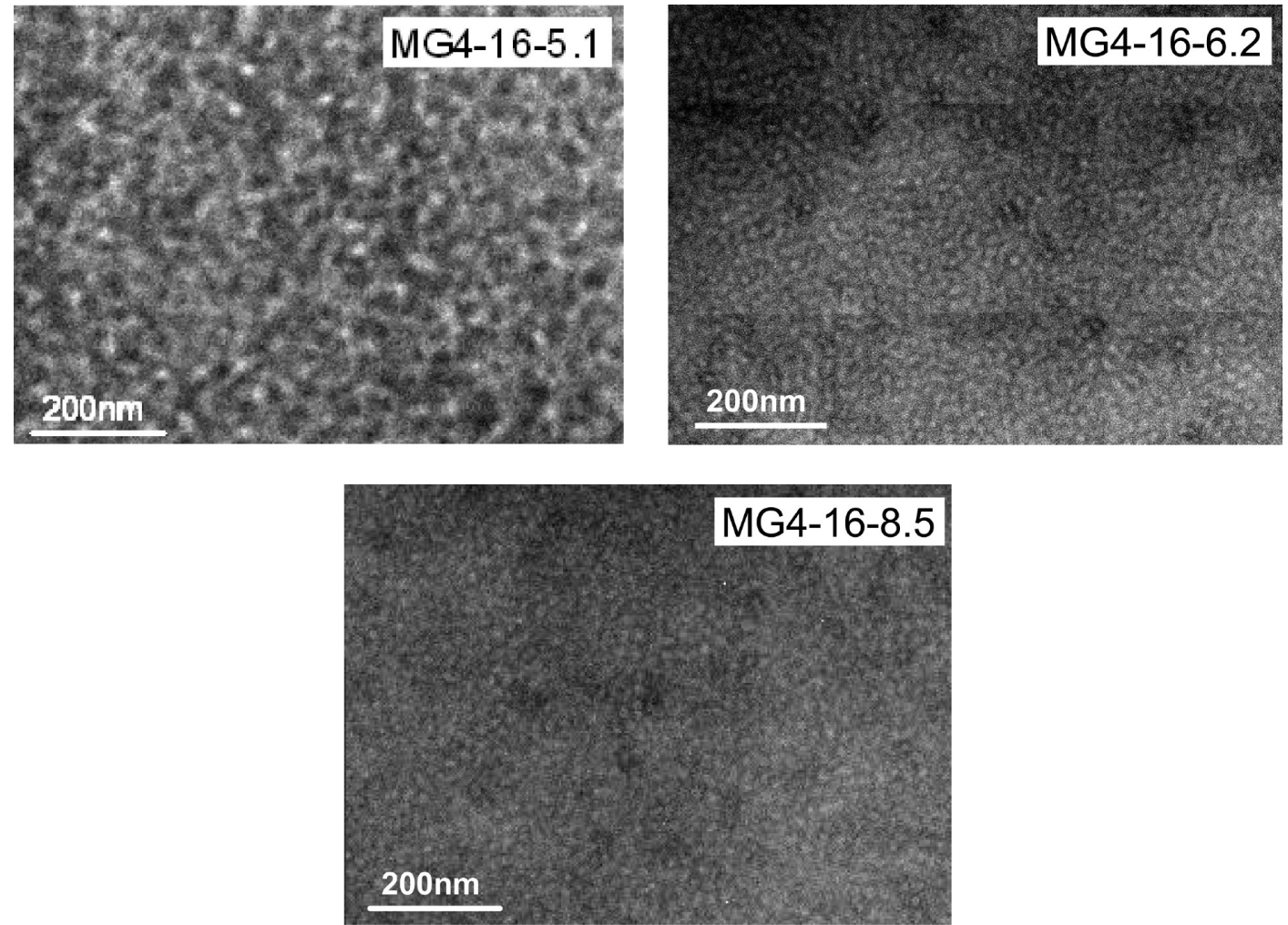

Figure 2. TEM images of tetrafunctional MG copolymers with $16 \mathrm{wt} \% \mathrm{PS}$.

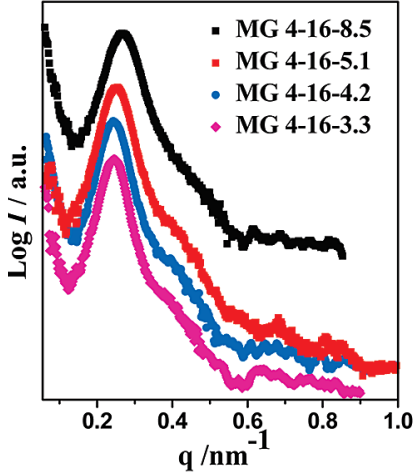

(a)

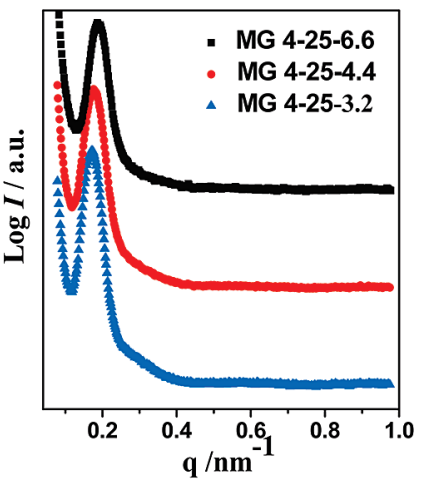

(b)
Figure 3. SAXS profiles of MG copolymers: (a) MG4-16 and (b) MG 4-25.

machine (Zwick 1456) and the specimen size according to ISO 527 type $5 \mathrm{~A}$. To ensure good measurement statistics, 5-10 tensile specimens were prepared for each sample by stamping the dogbone shape from cast and annealed polymer films.

\section{Results and Discussion}

1. Morphology. Figure 2 shows TEM micrographs of MG 4-16 with different numbers of branch points. It can be found that the morphology of tetrafunctional MG copolymers with 16 wt \% PS does not show significant dependence on $\beta$. One can see that all of materials have PS spheres dispersed in PI matrix. TEM investigation of other tetrafunctional MG copolymers with same PS content showed similar phase separated structure. The local microstructure observed by TEM was reconfirmed by SAXS investigation in the bulk samples. ${ }^{28}$ As shown in Figure 3a, the SAXS profiles indicate that all of the MG 4-16 samples have a microphase-separated structure, and the domain spacing calculated according to SAXS data has a very slightly decrease with increasing $\beta$. The domain spacing is $25 \mathrm{~nm}$ for MG 4-16-3.3 and $22.7 \mathrm{~nm}$ for MG 4-16-8.5. Such morphology investigation reveals that the PS phase is well dispersed in MG copolymers with higher $\beta$ value, and the continuity or integrity of PS domains decreases slightly with increasing $\beta$.

SAXS profiles of MG 4-25 are shown in Figure 3b. It could be found that all the samples have a strong primary peak which indicates a microphase-separated structure with a spacing of around $35.1 \mathrm{~nm}$. In addition, a very weak and broad second peak is observed for MG 4-25-3.2. This reveals that the MG 4-25 with low number of branch points has a well-ordered mirophase-separated structure. TEM investigation reveals the morphologies of $\mathrm{MG} \mathrm{4-25}$ have strong dependence on $\beta$. As presented in Figure 4, MG 4-25-3.2 forms long (hundreds of nanometers) and regularly packed PS cylinders, while disordered wormlike PS microdomains (tens of nanometers in length) are found for MG 4-25 with 4.4 and 6.6 branch points. It suggests that the size and intergrity of PS microdomains decrease with increasing unmber of branch point.

2. Deformation Mechanisms 2.1. Synchrotron $S A X S$. The 2-D SAXS patterns of deformed MG copolymers at different strain stages are shown in Figure 5. In order to fix the sample tightly between two clamps, some prestrain or prestress was introduced. So the 2-D SAXS pattern at $0 \%$ of strain was not captured. But from static SAXS measurement one can find that all of the investigated samples have a circular first-order 2-D SAXS pattern, which indicates the presence of phaseseparated morphology.

Figure 5a displays the 2-D SAXS patterns of MG 4-16-8.5 at different strain stages. This material has a spherical morphology at the beginning as shown by the TEM micrograph in Figure 2 and found in our earlier publication. ${ }^{6}$ Upon stretching, the initial circular scattering pattern becomes elliptical with its major axis perpendicular to the stretching direction (SD). With increasing strain, 

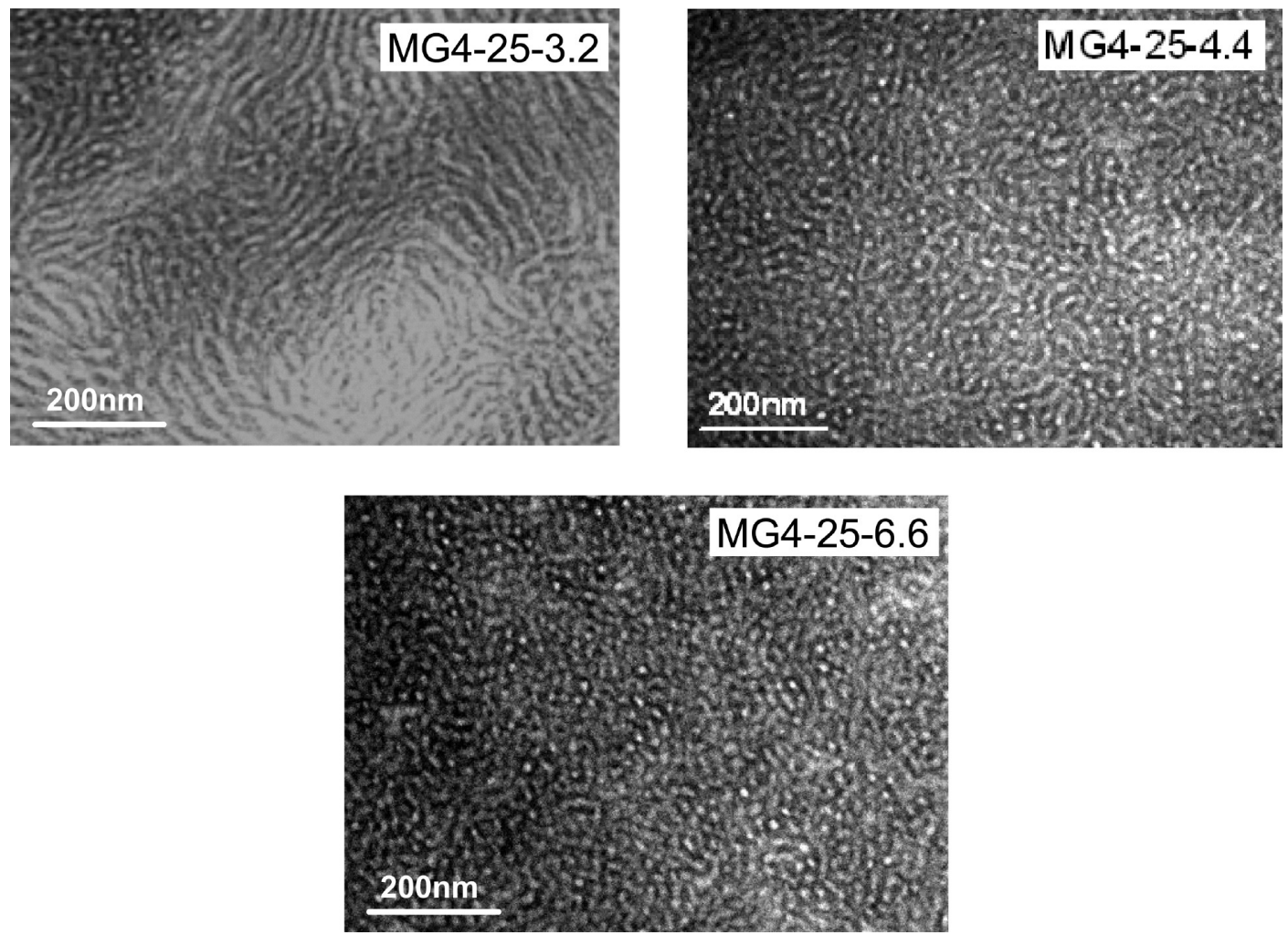

Figure 4. TEM images of tetrafunctional MG copolymers with 25 wt \% PS.

the scattering along SD, which is related to the microdomains originally oriented perpendicular to SD, moves toward the origin and the scattering perpendicular to SD, which is related to the microdomains originally orientated parallel to the SD, and moves away from the origin. The systematic variation of 2-D SAXS patterns indicates that the average interdomain distance along SD increases with a simultaneous decrease of that in the direction perpendicular to SD.

2-D SAXS patterns of MG 4-25-3.2 recorded at different strains are presented in Figure 5b. At low strain region, the evolution of SAXS patterns of MG 4-25-3.2 is similar to that of MG 4-16-8.5. But from 43\% strain, a four-point pattern was observed, which indicates that a tilted structure was formed with respect to SD. The angle $(\phi)$ in the four-point pattern (Figure 6a) can be related to the inclination angle $\left(90^{\circ}-\phi\right)$ of cylinders with respect to SD (Figure 6b). Inspection of Figure $5 \mathrm{~b}$ found that the four-point pattern angle increases with further increase of strain. This suggests that most of cylindrical microdomains inclined to SD and the angle between cylinders and SD decreases with increasing strain. From $81 \%$ strain, the maximal scattering in the four four-point pattern becomes weaker and disappears gradually with increasing strain, which may indicate the fracture of cylindrical PS microdomains. The 2-D SAXS pattern of deformed MG 4-25-6.6 at different strains is shown in Figure 5c. Upon stretching, the initial circular scattering pattern becomes elliptical with its major axis parallel to the meridian and its minor axis parallel to SD. The morphological scattering is much weaker than that in case of MG 4-25-3.2 because of the lower morphological ordering in MG 4-25-6.6 as shown by the TEM micrographs in Figure 4. Another difference between MG 4-25-3.2 and MG 4-25-6.6 is that the four-point pattern which is observed during stretching of MG 4-25-3.2 does not appear in MG 4-25-6.6.
According to the evolution of the first-order diffraction, the microstrain $\left(\varepsilon_{\mathrm{mi}}\right)$, which is also called the Bragg spacing strain, can be calculated with $\varepsilon_{\mathrm{mi}}=\left(d-d_{0}\right) / d_{0}$, where $d_{0}$ is the interdomain spacing of the original sample and $d$ is that of the deformed sample in stretching direction. In Figure 7 microstrain is plotted as a function of macrostrain. Affine deformation was found for all the investigated MG copolymers except MG 4-25-3.2. The data point of MG 4-25-3.2 at 120\% strain has an obvious deviation from affine deformation, which may be caused by the fracture of PS cylinders.

2.2. Rheo-optical FT-IR. In order to study the deformation mechanisms of MG copolymers at the molecular level, infrared dichroism analysis is employed. Figure 8 shows the polarized FT-IR spectra of MG 4-16-3.3 without deformation and at $1500 \%$ of strain. For the sample without deformation, no obvious difference can be observed between the spectra obtained with the direction of the polarized radiation parallel $(I I)$ and perpendicular $(\perp)$ to SD. But for the sample at $1500 \%$ of strain, the spectra collected with different polarization directions are showing small but obvious differences. The collective deformation from the heterogeneous phases is characterized by observing the selective band in individual phases. The absorption bands at 1376 and $1493 \mathrm{~cm}^{-1}$ (enlarged in Figure 8) are selected for PI and PS phases, respectively. The band at $1376 \mathrm{~cm}^{-1}$ is assigned to the symmetric vibration of $\mathrm{CH}_{3}$, while the band at $1493 \mathrm{~cm}^{-1}$ can be assigned to a $\mathrm{C}-\mathrm{H}$ in plane stretching mode. The transition moments of both bands are assumed to be about $90^{\circ}$ with respect to PI and PS chain axes, respectively. It can be observed that, for the sample at $1500 \%$ of strain, both the bands at 1376 and $1493 \mathrm{~cm}^{-1}$ have higher absorbance in the spectrum with the polarization perpendicular to SD. Qualitatively, this observation suggests that both PI and PS molecular chains are oriented along SD. 


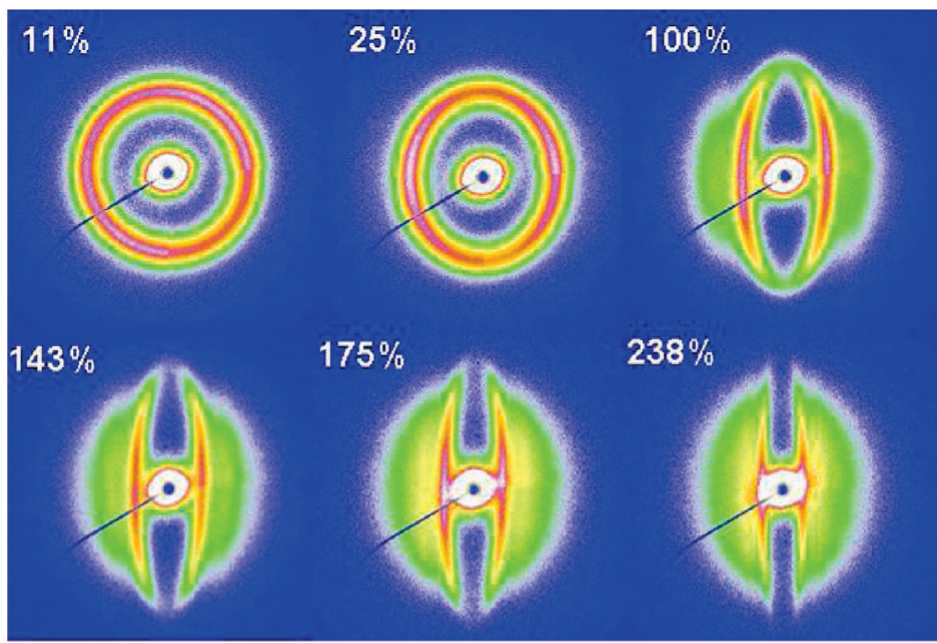

(a)

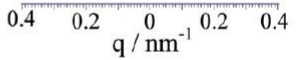

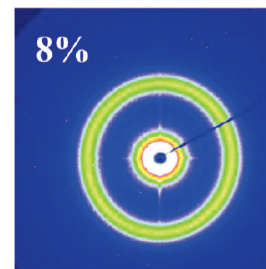

\begin{abstract}
$39 \%$
\end{abstract}

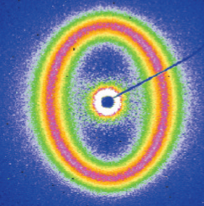

$81 \%$

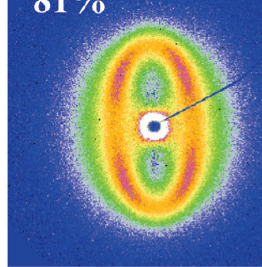

$116 \%$

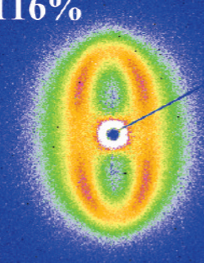

$43 \%$

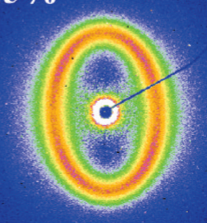

$164 \%$

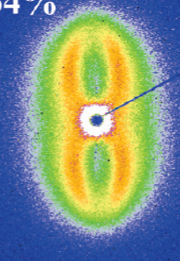

$70 \%$

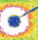

(b)
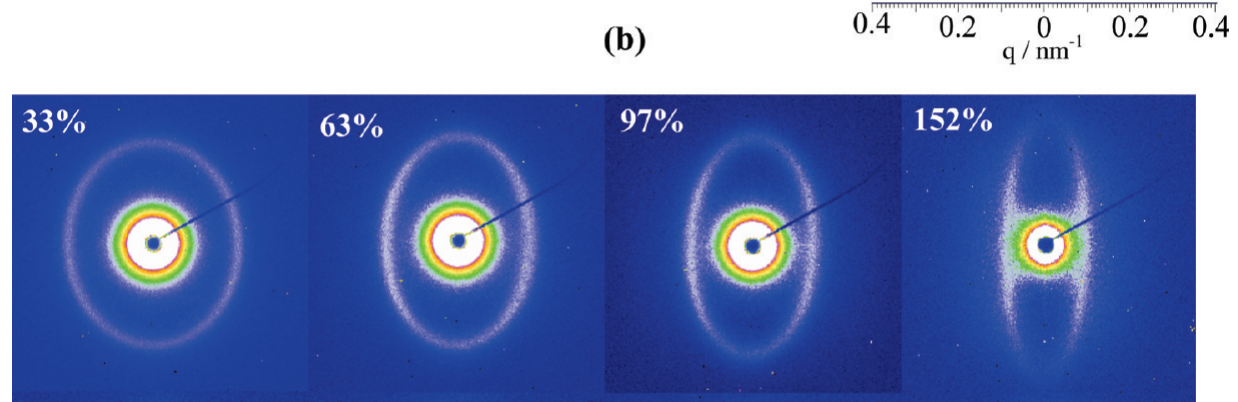

$177 \%$

$225 \%$

$63 \%$

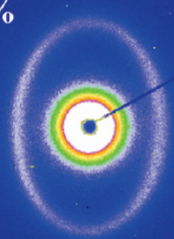

$97 \%$

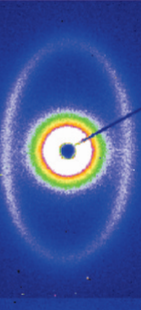

$251 \%$

$333 \%$

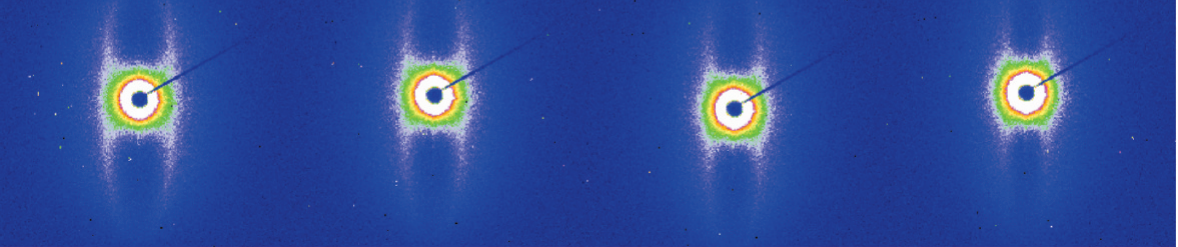

(c)

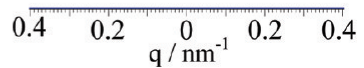

Figure 5. Evolution of 2-D SAXS patterns with strain: (a) MG 4-16-8.5, (b) MG 4-25-3.2, and (c) MG 4-25-6.6. The stretching direction is horizontal.

Quantitative analysis of the infrared dichroism provides more information on the orientation behavior of PI and PS chains during the stretching process. The infrared dichroism allows one to determine the orientation function (eq 1). Here, $R$ represents the dichroic ratio which is given by $R=A_{\|} / A_{\perp}$ (where with $A_{\|}$and $A_{\perp}$ are the absorbances of specific absorption bands measured with IR radiation polarized parallel and perpendicular to the $\mathrm{SD}$, respectively), and $R_{0}=2 \cot ^{2} \psi$, where $\psi$ is the angle between the chain axis and the transition moment 


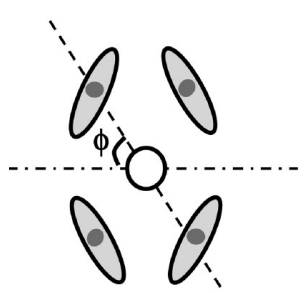

(a)

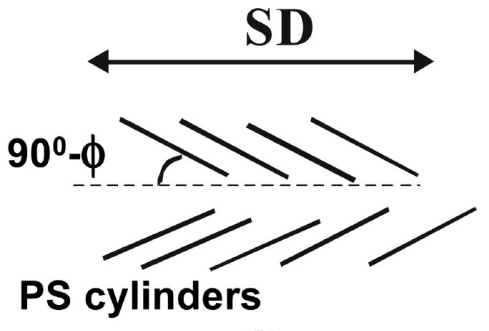

(b)
Figure 6. Schematic representation showing the relation between the angle of four-point 2-D SAXS pattern and the orientation of PS cylinders with respect to the stretching direction: (a) four-point 2-D SAXS pattern; (b) schematic diagram showing the orientation of PS cylinders with respect to SD.

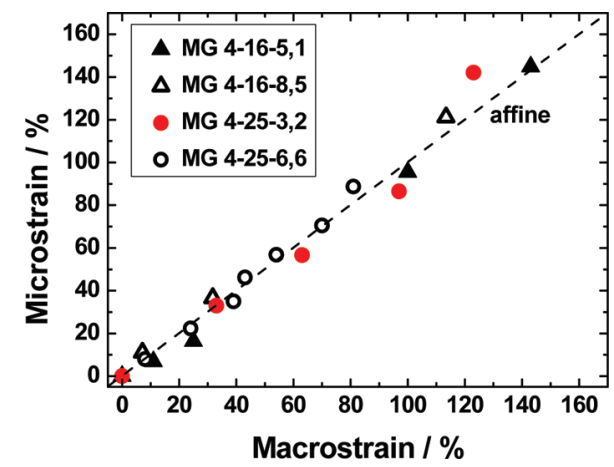

Figure 7. Microstrain plotted as a function of macrostrain.

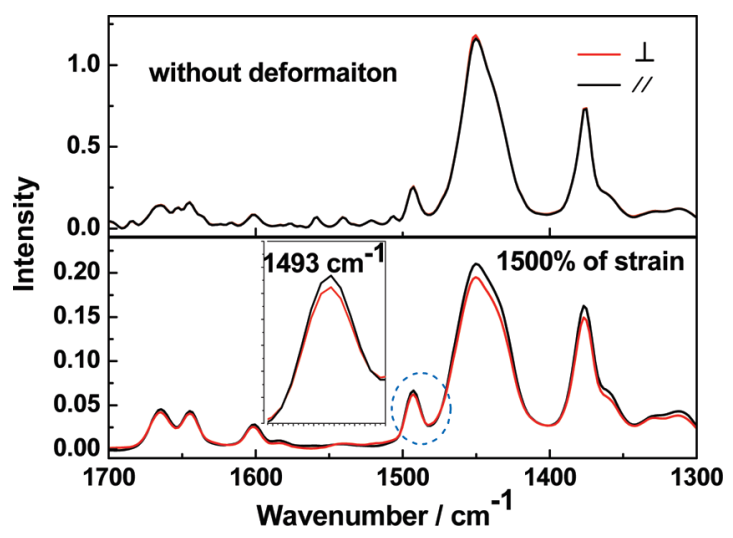

Figure 8. Polarized FT-IR polarization spectra of MG 4-16-3.3.

of the infrared vibration under consideration.

$$
f=\frac{\left(R_{0}+2\right)(R-1)}{\left(R_{0}-1\right)(R+2)}
$$

For the infrared vibration with a transition moment parallel to the chain axis, eq 1 can be written as eq 2 . When the transition moment of the infrared vibration is perpendicular to the chain axis, eq 3 can be obtained.

$$
\begin{gathered}
f=\frac{R-1}{R+2} \\
f=-2 \frac{R-1}{R+2}
\end{gathered}
$$

An increase of orientation function value $(f)$ corresponds to an increase in orientation and vice versa. The transition moments of both bands at 1376 and $1493 \mathrm{~cm}^{-1}$ are assumed to be about $90^{\circ}$ with respect to the PI and PS chain axes, respectively. Thus, eq 3 was applied, and Figure 9a,b shows the orientation function value of selected bands vs strain for MG 4-16-5.1 and MG 4-16-3.3, respectively. One can see that for MG 4-16-3.3 at smaller strains, i.e., below $500 \%$ of strain, the $f$ value of $1376 \mathrm{~cm}^{-1}\left(f_{\mathrm{PI}}\right)$ and $1493 \mathrm{~cm}^{-1}\left(f_{\mathrm{PS}}\right)$ show similar trends in their strain dependence and increase with increasing strain. At higher elongations, $f_{\mathrm{PI}}$ continues increasing, but $f_{\mathrm{PS}}$ shows only a very slight increase with increasing strain. However, it can be observed that even at quite high strains, i.e., above $1500 \%$, the values of both $f_{\mathrm{PI}}$ $(0.13)$ and $f_{\mathrm{PS}}(0.07)$ are comparable and quite small when compared to the value for the perfect orientation $(f=1)$. This indicates that upon stretching both PS and PI segments are only aligned slightly along SD. The lower orientation function value for the PI component with respect to the perfect orientation in MG copolymers has been observed in our earlier publication ${ }^{23}$ and discussed in detail. The $f_{\mathrm{PI}}$ and $f_{\mathrm{PS}}$ for MG 4-16-5.1 have almost same dependence on strain except that $f_{\mathrm{PS}}$ increases a little slower than $f_{\mathrm{PI}}$ from $1000 \%$ strain. The evolution of the orientation function value of the selected bands for MG 4-25-3.2 is shown in Figure $9 \mathrm{c}$. The stronger orientation in PI than that in the PS phase can be more clearly noticed in $\mathrm{MG} \mathrm{4-25-3.2.} f_{\mathrm{PS}}$ increases only slightly, whereas $f_{\mathrm{PI}}$ increases fast with increasing strain.

A combined analysis of synchrotron SAXS and rheooptical FR-IR measurements offers the opportunity to deduce deformation mechanisms for MG copolymers. For MG 4-16, the special molecular architecture and morphology make PS spheres highly ductile and mobile. Upon stretching, both phases are deformed, and the molecular chains of both components are oriented along SD. Further elongation of the material causes breakage. The analysis on the relation between microstrain and macrostrain suggests that the deformation is affine (Figure 7) in the investigated deformation region. MG 4-25 is also deformed affinely in the low stain range. At low strain, the material is elongated homogeneously with increasing strain; a four-point 2-D SAXS pattern is observed for MG 4-25-3.2. This implies that most of PS cylinders assume a preferential orientation inclined to SD with some angle, and the angle decreases with increasing strain. Decreasing of the intensity of maxima in the four-point pattern at higher elongation indicates that PS cylinders suffer from fracture. For MG 4-25-6.6, no obvious four-point 2-D SAXS pattern was observed. This is because the large number of branch points prevents the formation of ordered phase structures. Upon stretching, the wormlike PS microdomains in MG 4-25-6.6 may also tend to rotate with respect to SD. However, because of the higher disorder, PS micordomains cannot rotate co-operatively, and the angles between individual PS microdomains and SD are very different. So no obvious four-point pattern was observed. This reveals that the strain-induced orientation of the PS phase is at different dimension scales for MG copolymers with different morphologies. For cylinder forming MG 4-253.2 , the PS phase has a higher integrity; the appearance of the four-point SAXS pattern indicates that the orientation of individual PS microdomain as a unity and the co-operative orientation of different PS microdomains. For sphere-forming MG 4-16 with more ductile PS phase, an individual PS molecular chain has obvious orientation along SD. For all investigated MG copolymers, PI phase has strain-induced orientation along SD at the molecular scale.

3. Tensile Properties. Parts a and b of Figure 10 show the stress-strain curves of MG 4-16 and MG 4-25, respectively. 


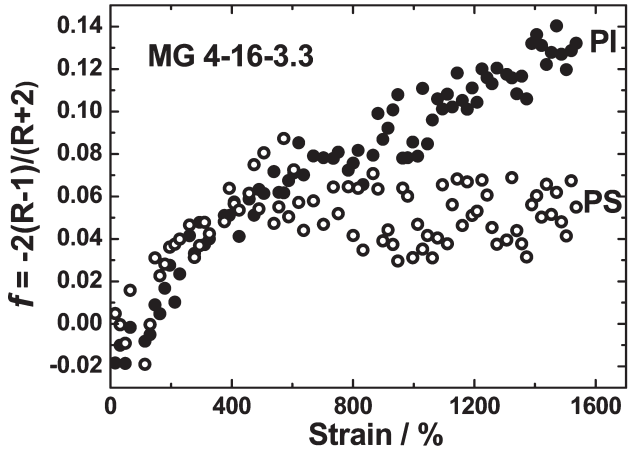

(a)

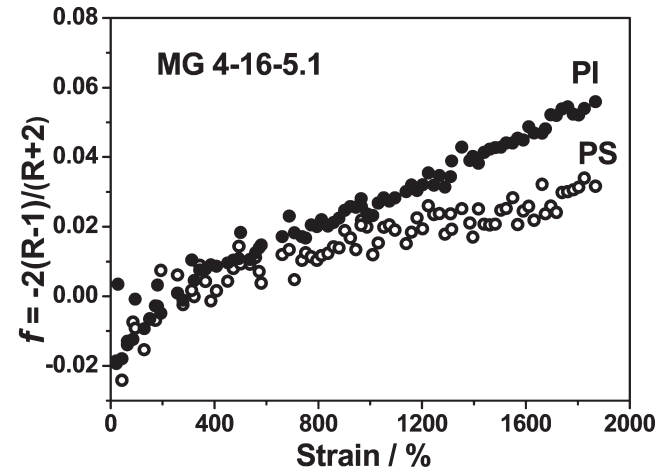

(b)

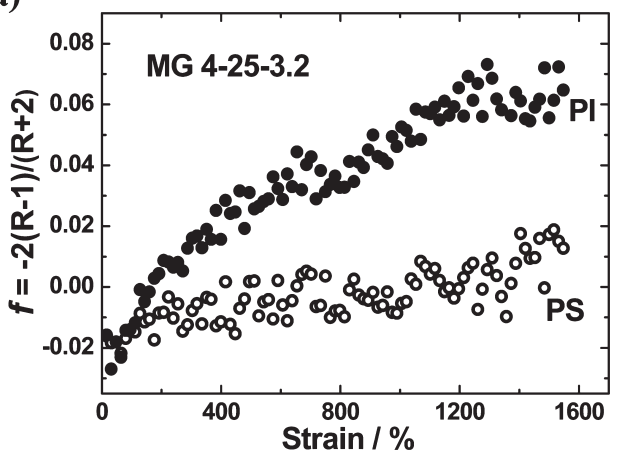

(c)

Figure 9. Strain dependence of the orientation function for the PI and PS component: (a) MG 4-16-5.1, (b) MG 4-16-3.3, and (c) MG 4-25-3.2.

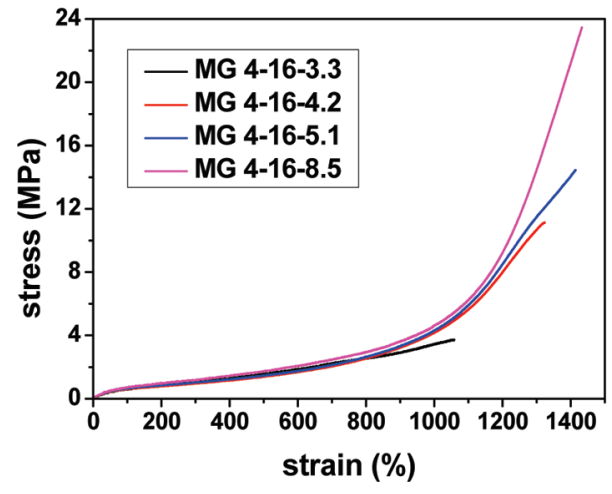

(a)

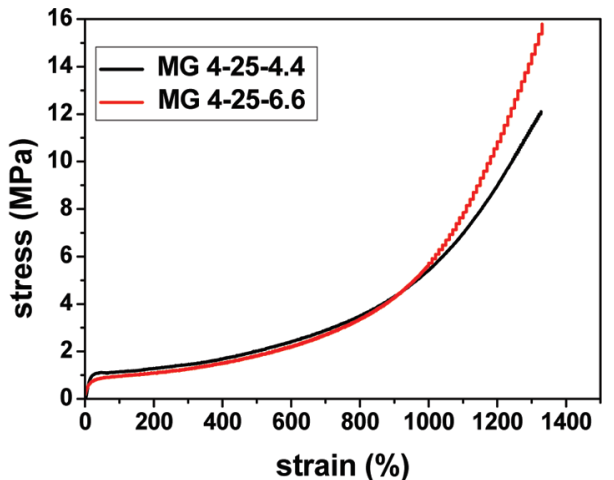

(b)

Figure 10. Stress-strain curves of tetrafunctional MG copolymers: (a) MG 4-16 and (b) MG 4-25.

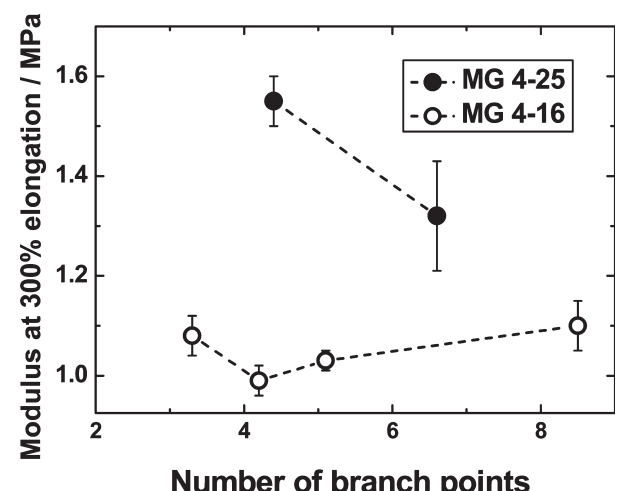

Figure 11. Influence of the number of branch points on modulus at $300 \%$ elongation. The dashed lines are a guide to the eye.

Most obvious is that the tensile strength increases with increasing number of branch point $(\beta)$ for both series of tetrafuncitonal MG copolymers. Careful inspection finds that, for MG 4-16, all the tensile curves almost overlap in the low-medium stretching region, which means that tensile properties of MG 4-16 has no obvious dependence on $\beta$ in this region. Whereas for $\mathrm{MG} 4-25$, tensile properties at low-medium range have small but obvious difference with increasing $\beta$.

Figure 11 shows the modulus at $300 \%$ elongation or $300 \%$ modulus (defined as the stress required to obtain $300 \%$ strain in rubber industry) of MG copolymers as a function of the number of junction points per molecule $(\beta)$. For tetrafunctional MG copolymers with 16 wt $\%$ PS, the modulus at $300 \%$ elongation is nearly independent on $\beta$, whereas it decreases with increasing $\beta$ for MG copolymers with $25 \mathrm{wt} \%$ PS.

The different correlation between modulus and $\beta$ for tetrafunctional MG copolymers with different PS content can be interpreted from the view of the molecular architecture and PS domain continuity. As found in the section of morphology, all of MG 4-16 samples have similar microphase-separated structure, and the domain spacing 
of MG 4-16 decreases slightly with increasing $\beta$. Such morphology investigation reveals that the PS phase is better dispersed in MG copolymers with higher $\beta$ value, and the continuity or integrity of PS domains decreases slightly with increasing $\beta$, which will decrease the elastic modulus. On the other hand, as shown in Figure 1 and interpreted in our earlier publication, ${ }^{23}$ there are two kinds of PI blocks in MG copolymers: PI intermediate blocks and PI end blocks. The PI blocks in all investigated MG copolymers may be assumed to be well-entangled, i.e., $M_{\mathrm{PI} \text { block }}>2 M_{\mathrm{e}, \mathrm{PI}}\left(M_{\mathrm{e}, \mathrm{PI}} \sim 4 \mathrm{~kg} /\right.$ mol). ${ }^{29}$ However, in case of PI intermediate blocks, the entangled PI chains are pinned by the glassy PS domains at both ends, whereas PI end blocks are only tethered at one end by the glassy domains. The increase of the proportion of PI intermediate blocks with increasing $\beta$ contributes to the elastic behavior because PI intermediate blocks form trapped entanglements and have less mobility than the PI end blocks. The influence from the continuity of PS domains and the proportion of PI intermediate blocks seems to counteract, and the elastic modulus of MG 4-16 has no obvious dependence on $\beta$.

The morphologies of MG 4-25 have a strong dependence on $\beta$. The size and integrity of PS phase decrease dramatically with increasing $\beta$. The higher continuous PS microdomains can bear more initial stress. Decreasing of continuity and size of PS microdomains increases the interface between PS and PI phases. This leads to more polymer segments located in the interface area and makes the PS phase more flexible because styrene segments in the vicinity of the interface will have a greater degree of mobility than those in the interior. ${ }^{30,31}$ So the modulus decreases with increasing $\beta$. The proportion of intermediate PI spacers also increases with increasing $\beta$, but it seems that the dominant factor is the continuity of PS microdomains. Qiao et al. ${ }^{32}$ studied the mechanical properties of a single SIS triblock copolymer with different morphologies by casting with solvents of varying selectivity to PS and PI. It was also found that the elastic moduli increased with increasing glassy domain continuity.

The different correlations between modulus and $\beta$ for tetrafunctional MG copolymers with different PS content can also be well related to the orientation behavior of PS and PI molecular chains detected by infrared dichroism. For cylinder-forming MG copolymers, the PS phase is much more rigid than the PI phase and can bear much initial loading, so $f_{\mathrm{PS}}$ shows only a slight increase with deformation and the materials exhibit higher elastic modulus compared to sphere-forming tetrafunctional MG copolymers. For sphere-forming tetrafunctional MG copolymers, the fast increase of $f_{\mathrm{PI}}$ and $f_{\mathrm{PS}}$ at the low-strain region suggests that the PS phase is ductile and does not bear much of the initial loading, which results in the lower modulus. At the highstrain region most of PS chains located in the interface area have been stretched and the PS chains in the interior of the PS phase are more rigid so the nominal change of $f_{\mathrm{PS}}$ is observed. The strong morphological dependence of the molecular orientation behavior of the PS-based copolymer has been found in recent studies. ${ }^{33-35}$

Another point in the context of the modulus is that tetrafunctional MG copolymers with $16 \mathrm{wt} \%$ PS have a lower modulus than those with $25 \mathrm{wt} \%$ PS. This is quite easy to understand since the former has lower PS content and its PS domains have lower continuity.

The influence of the number of junction points on tensile strength (stress at break) of tetrafunctional MG copolymers is presented in Figure 12. One can see that, for tetrafunctional MG copolymers with given PS content, tensile

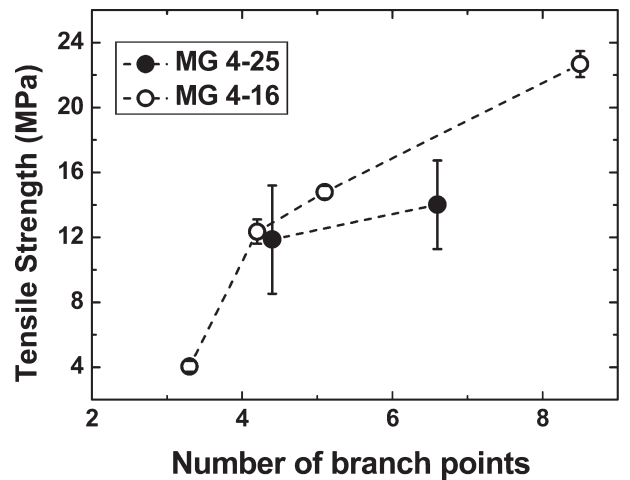

Figure 12. Influence of the number of branch points on the tensile strength. The dashed lines are a guide to the eye.

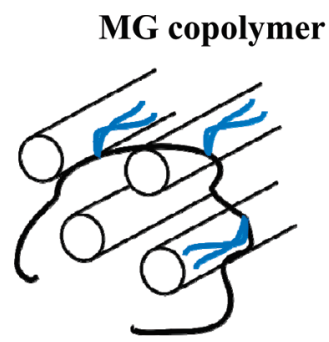

PS

\section{SIS copolymer}

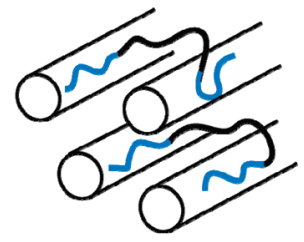

PI
Figure 13. Schematic diagram showing the difference between tetrafunctional MG copolymers and SIS with PS cylinders dispersed in the PI matrix. In MG copolymers one PI block can be tethered with a large number of PS domains; by contrast, one PI block in a linear SIS can bridge only two PS cylinders.

strength increases with increasing $\beta$. An increase in $\beta$ can decrease the continuity and size of PS mircodomains and improve the proportion of PI blocks bridging PS microdomains. But at high strain, the difference in the continuity and size of PS microdomains decreases because most of PS microdomains are fragmented into small particles, which has been verified by the strain related 2-D SAXS patterns in Figure $5 \mathrm{~b}$ and confirmed by numerous studies. ${ }^{11,33,36-38}$ Therefore, the initial morphology is not dominant in control of the deformation behavior at high elongation. The PI blocks bridging PS microdomains can bear stress more effectively than the dangling PI end blocks, and the proportion of PI blocks bridging PS microdomains is dominant on the tensile strength. Another important result shown in Figure 12 is that, at given number of branch points, the tensile strength of MG copolymer with $16 \mathrm{wt} \% \mathrm{PS}$ is slightly higher than MG copolymer with $25 \mathrm{wt} \% \mathrm{PS}$. This is surprising because for general PS-based linear block copolymers, the tensile strength increases with increasing PS content. ${ }^{39}$ Such observations may be attributed to the better dispersion of PS domains in MG 4-16, which can distribute the applied stress more uniformly and result in more effective stress transfer between PI and PS phases. And the molecular weight of PI blocks in MG 4-16 and MG 4-25 is 97.2 and 87.6 $\mathrm{kg} / \mathrm{mol}$, respectively. The higher molecular weight of PI blocks in MG 4-16 may also contribute more to the tensile strength.

4. Origin of Superelasticity. The most exceptional property of the investigated MG copolymers is their exceptionally high strain at break and low residual strain, which we named superelasticity. ${ }^{4,5}$ In one of our recently published papers ${ }^{23}$ we interpreted that the superelasticity of 
trifunctional MG copolymers as originating from the doubled functionality of PS microdomains when compared to SIS triblock copolymers. Another important contribution from the special molecular structure of $\mathrm{MG}$ copolymers is the possibility for the entropic PI springs to be tethered with a large number of PS domains. As shown in Figure 13, in SIS the PI block can bridge only two PS domains. In contrast, in MG copolymers the elastic PI backbone can more effectively couple to a larger number of reinforcing PS domains and in this way more effectively communicate structural and mechanical rigidity among neighboring domains. This may increase the elasticity of MG copolymers at high strains and improve the strain at break.

\section{Conclusion}

From a combined study of structure and mechanical aspect of MG copolymers with different architecture, it was revealed that the number of branch points has no obvious influence on the microphase-separated structure of tetrafunction MG copolymers with 16 wt \% PS. But for tetrafunctional MG copolymers with $25 \mathrm{wt} \% \mathrm{PS}$, the size and integrity of PS mircodomains decrease with increasing number of branch point. The deformation mechanism of $\mathrm{MG}$ copolymers is highly related to the morphology. For sphere-forming MG 4-16, both phases are deformed and the molecular chains of both components are oriented along the SD. For MG 4-25-3.2 with ordered PS cylinders dispersed in the PI matrix, upon stretching, most of PS cylinders assume a preferential orientation inclined to the SD at an angle which decreases with increasing strain. At higher elongation, the PS microdomain suffer from fracture. In conclusion, dependent on the phase-separated morphology and integrity of PS phase, the strain-induced orientation of PS phase is at different dimension scales. Further analysis on the tensile properties, morphology, and deformation behavior found that the elastic modulus is controlled by the continuity of PS microdomains, while the proportion of PI blocks effectively bridging PS domains controls the deformation of MG copolymers at high strain. More continuous PS domains can share a greater portion of the initial load, which results in the higher modulus values. For cylinder-forming tetrafunctional MG copolymers, the size and continuity of the PS phase decrease dramatically with increasing number of branch points $(\beta)$. So the modulus at $300 \%$ elongation decreases with increasing $\beta$. But for sphere-forming tetrafunctional MG copolymers, the size of PS spheres decreases slightly, and the proportion of PI intermediate blocks increases with increasing $\beta$. The influence from the continuity of PS domains and the proportion of PI intermediate blocks counteract and the elastic modulus of MG 4-16 has no obvious dependence on $\beta$. In the high-strain region, most of PS domains are fragmented and the influence from the continuity of PS microdomains decreases. The tensile strength is controlled by the proportion of intermediate bridging PI blocks and increases with increasing $\beta$ for both series of tetrafunctional MG copolymers. The special molecular architecture and thus-obtained higher effective functionality of PS domains and higher possibility for one long PI backbone chains to be tethered with large number of PS microdomains are the origin of the superelasticity for MG copolymers.

Acknowledgment. Y. X. Duan is grateful to the Alexander von Humboldt foundation for kindly providing the fellowship in Germany which made it possible for her to perform this study. J. Mays acknowledges support through the Division of Materials Science and Engineering, Office of Basic
Energy Sciences, U.S. Department of Energy (DE-ACo500OR22725).

\section{References and Notes}

(1) Mays, J. W.; Uhrig, D.; Gido, S. P.; Zhu, Y. Q.; Weidisch R.; Iatrou, H.; Hadjichristidis, N.; Hong, K.; Beyer, F. L.; Lach, R.; Buschnalowski, M. Macromol. Symp. 2004, 215, 111126.

(2) Iatrou, H.; Mays, J. W.; Hadjichristidis, N. Macromolecules 1998, 31, 6697-6701.

(3) Uhrig, D.; Mays, J. W. Macromolecules 2002, 35, 7182-7190.

(4) Weidisch, R.; Gido, S. P.; Uhrig, D.; Iatrou, H.; Mays, J. W.; Hadjichristidis, N. Macromolecules 2001, 34, 6333-6337.

(5) Staudinger, U.; Weidisch, R.; Zhu, Y.; Gido, S. P.; Uhrig, D.; Mays, J. W.; Iatrou, H.; Hadjichristidis, N. Macromol. Symp. 2006, 233, 42-50.

(6) Zhu, Y. Q.; Burgaz, E.; Gido, S. P.; Staudinger, U.; Weidisch, R.; Uhrig, D.; Mays, J. W. Macromolecules 2006, 39, 4428-4436.

(7) Beyer, F. L.; Gido, S. P.; Buschl, C.; Iatrou, H.; Uhrig, D.; Mays, J. W.; Chang, M. Y.; Garetz, B. A.; Balsara, N. P.; Tan, N. B.; Hadjichristidis, N. Macromolecules 2000, 33, 2039-2048.

(8) Hashimoto, T.; Fujimura, M.; Saijo, K.; Kawai, H.; Diamant, J.; Shen, M. In Multiphase Polymers; Cooper, S. L., Estes, G. M., Eds.; Advances in Chemistry Series 176; American Chemical Society: Washington, DC, 1979; p 257.

(9) Inoue, T.; Moritani, M.; Hashimoto, T.; Kawai, H. Macromolecules 1971, 4, 500-507.

(10) Pakula, T.; Saijo, K.; Kawai, H.; Hashimoto, T. Macromolecules 1985, 18, 1294-1302.

(11) Polizzi, S.; Bosecke, P.; Stribeck, N.; Zachmann, H. G.; Zietz, R.; Bordeianu, R. Polymer 1990, 31, 638-645.

(12) Prasman, E.; Thomas, E. L. J. Polym. Sci., Part B: Polym. Phys. 1998, 36, 1625-1636.

(13) Cohen, Y.; Albalak, R. J.; Dair, B. J.; Capel, M. S.; Thomas, E. L. Macromolecules 2000, 33, 6502-6516.

(14) Honeker, C. C.; Thomas, E. L.; Albalak, R. J.; Hajduk, D. A.; Gruner, S. M.; Capel, M. C. Macromolecules 2000, 33, 9395-9406.

(15) Séguéla, R.; Prud'homme, J. Macromolecules 1988, 21, 635-643.

(16) Séguéla, R.; Prud'homme, J. Macromolecules 1978, 11, 1007-1016.

(17) Séguéla, R.; Prud'homme, J. Macromolecules 1981, 14, 197-202.

(18) Zhao, Y. Macromolecules 1992, 25, 4705-4711.

(19) Takahashi, Y.; Song, Y.; Nemoto, N.; Takano, A.; Akazawa, Y.; Matsushita, Y. Macromolecules 2005, 38, 9724-9729.

(20) Huy, T. A.; Hai, L. H.; Adhikari, R.; Weidisch, R.; Michler, G. H.; Knoll, K. Polymer 2003, 44, 1237-1345.

(21) Sakurai, S.; Sakamoto, J.; Shibayama, M.; Nomura, S. Macromolecules 1993, 26, 3351-3356.

(22) Sakamoto, J.; Sakurai, S.; Doi, K.; Nomura, S. Polymer 1993, 34, 4837-4840.

(23) Duan, Y. X.; Rettler, E.; Schneider, K.; Schlegel, R.; Thunga, M.; Weidisch, R.; Siesler, H. W.; Stamm, M.; Mays, J. W.; Hadjichristidis, N. Macromolecules 2008, 41, 4565-4568.

(24) Schneider, K.; Trabelsi, S.; Zafeiropoulos, N. E.; Davies, R.; Riekel, C.; Stamm, M. Macromol. Symp. 2006, 236, 241-248.

(25) Davies, R. J.; Zafeiropoulos, N. E.; Schneider, K.; Roth, S. V.; Burghammer, M.; Riekel, C.; Kotek, J. C.; Stamm, M. Colloid Polym. Sci. 2004, 282, 854-866.

(26) Siesler, H. W. Makromol. Chem., Macromol. Symp. 1992, 53, 8995.

(27) Siesler, H. W.; Hoffmann, G. G.; Kolomiets, O.; Pfeifer, F.; Zahedi, M. In Vibrational Spectroscopy of Polymers: Principles and Practice; Everall, N. J., Chalmers, J. M., Griffiths, P. R., Eds.; John Wiley \& Sons Ltd.: Chichester, UK, 2007; pp 313-347.

(28) Thunga, M.; Schlegel, R.; Staudinger, U.; Duan, Y. X.; Weidisch, R.; Heinrich, G.; Mays, J. W.; Hadjichristidis, N. Kautschuk Gummi Kunststoffe 2008, 11, 597-605.

(29) Fetters, J. J.; Lohse, D. J.; Richter, D.; Witten, T. A.; Zirkel, A. Macromolecules 1994, 27, 4639-4647.

(30) Hutchison, J. L.; Samulski, E. T.; Smith, S. D. Polym. Prepr. (Am. Chem. Soc., Div. Polym. Chem.) 1993, 34, 618-620.

(31) Smith, D. D.; Noda, I.; Marcott, C.; Dowrey, A. E.; Russel, T. P.; Mayes, A.; Kornfield, J. A.; Chung, C. C.; Samulski, E. T.; Hutchison, J. Polym. Prepr. (Am. Chem. Soc. Div. Polym. Chem.) 1994, 35, 96-99.

(32) Qiao, L.; Leibig, C.; Hahn, S. F.; Winey, K. I. Ind. Eng. Chem. Res. 2006, 45, 5598-5602. 
(33) Adhikari, R.; Michler, G. H. Prog. Polym. Sci. 2004, 29, 949-986.

(34) Van Asselen, O. L. J.; Van Casteren, I. A.; Goossens, J. G. P.; Meijer, H. E. H. Macromol. Symp. 2004, 205, 85-94.

(35) Adhikari, R.; Hyu, T. A.; Buschnalowski, M.; Michler, G. H.; Knoll, K. New J. Phys. 2004, 6, 28.

(36) Odell, J. A.; Keller, A. Polym. Eng. Sci. 1977, 17, 544-559.
(37) Kwee, T.; Taylor, S. J.; Mauritz, K. A.; Sorey, R. F. Polymer 2005, 46, 4480-44891.

(38) Mclean, R. S.; Sauer, B. B. J. Polym. Sci., Part B: Polym. Phys. 1999, 37, 859-866.

(39) Morton, M.; Mcgrath, J. E.; Juliano, P. C. J. Polym. Sci., Part C 1969, 26, 99-115. 